\title{
OPTIMAL RECOVERY APPROACH TO IMAGE INTERPOLATION
}

\author{
D. Darian Muresan and Thomas W. Parks \\ School of Electrical and Computer Engineering, Cornell University \\ Ithaca, NY 14853 \\ darian,parks@ee.cornell.edu
}

\begin{abstract}
We consider the problem of image interpolation from an adaptive optimal recovery point of view. Many different standard interpolation approaches may be viewed through the prism of optimal recovery. In this paper we review some standard image interpolation methods and how they relate to optimal recovery as well as introduce a broader, more general and systematic approach to image interpolation using adaptive optimal recovery.
\end{abstract}

\section{INTRODUCTION}

Any successful image interpolation method requires a good image model. The standard bi-cubic interpolation assumes that the image is locally cubic, in [3] the assumption is that locally each pixel can be represented as a linear combination of the known neighboring pixels and in [2] we assumed that the image had been passed through a known low-pass filter, before decimation. One new category of interpolation algorithms is based on learning. In particular, the idea is to use the coarse scale image, or a collection of other similar images, to learn something about the given image and then use that knowledge to interpolate.

In this paper we give a brief review of the theory of optimal recovery $[5,7,8]$. We show how knowledge of the quadratic class can be used in deriving some common interpolation algorithms. Finally, we present a new and systematic method for learning the local quadratic signal class and show how to use this for image interpolation. This method is different and more systematically motivated than the method we presented in [1].

\section{OPTIMAL RECOVERY}

In this section we briefly review the theory of optimal recovery as applied to the interpolation problem [8]. We then apply this theory to develop a new adaptive approach to image interpolation. The interpolation problem may be viewed as a problem of estimating missing samples of an image.

This work was supported by NSF MIP9705349, TI and Kodak
This latter problem can be examined using the theory of optimal recovery. The theory of optimal recovery provides a broader setting, which illuminates the process of interpolation, by providing error bounds and allowing calculation of worst-case images which achieve these bounds.

Locally, at location $y$, we model the image as belonging to a certain ellipsoidal signal class $K$

$$
K=\left\{x \in R^{n}: x^{T} \mathbf{Q} x \leq \epsilon\right\}
$$

where $\mathbf{Q}$ is derived from the local image pixels or may be assumed known. Vector $x$ is any subset of the image containing the missing pixel $y$. Vector $x$ is chosen such that any $L$ linear functionals $\left(F_{i}, i=1, \ldots, L\right)$ of $x$ are assumed known. If we note the actual values of the functionals by $f_{i}$ we have $F_{i}(x)=f_{i}$. In this paper we assume that the functionals are based on derivatives and/or actual pixel values of the decimated image. The known functionals $F_{i}$, in the local image, determine a hyper-plane $\mathcal{X}$.

The intersection of the hyper-plane and ellipsoid is a hyper-circle in $\mathcal{X}$. The intersection depends upon the known functionals of the local image and we call it $C_{x}$. Formally,

$$
C_{x}=\left\{x \in \mathcal{X}: F_{i}(x)=f_{i},\|x\|_{Q} \leq 1\right\},
$$

For a linear mapping $\mathbf{U}$, the image of $C_{x}$ under $\mathbf{U}$ is the range of values that $\mathbf{U} f$ can take. The optimal recovery problem is to select the value in $\mathcal{X}$ which is a best approximation over all $\mathbf{U} f$ in $\mathbf{U} C_{x}$. We want to minimize

$$
\delta=\max _{x^{T} Q x}|\hat{y}-y|
$$

The Chebyshev center achieves this minimization. The Chebyshev center has been shown to be the minimum $Q$-norm signal on the hyper-plane determined by the known samples. The solution to this problem is well-known: see $[8,7]$.

If the collection of known functionals is $f_{i}$, the minimum norm signal is $\bar{u}$. Signal $\bar{u}$ is the unique signal in $\mathcal{X}$ with the property,

$$
\|\bar{u}\|_{Q}=\inf _{F_{i}(x)=f_{i}}\|x\|_{Q}
$$


Our estimates signal $\bar{u}$ must satisfy $F_{i}(\bar{u})=f_{i}$ and we are estimating $F(\bar{u})=f$. As shown in [8] there exist vectors $\phi, \phi_{1}, \ldots, \phi_{L}$ such that $F(\hat{u})=(\phi, \bar{u})_{Q}$ and

$$
F_{i}(\hat{u})=\left(\phi_{i}, \bar{u}\right)_{Q}
$$

where the parentheses denote a $\mathbf{Q}$ dot product. Vectors $\phi_{i}$ are known as the representers. From [8] the solution is given by

$$
\bar{u}=\sum_{i=1}^{L} \alpha_{i} \phi_{i}
$$

where the constants $\alpha_{i}$ are determined from the constraint of equation (4).

An advantage of this approach is not only that we can minimize the distance $\delta=\max _{x^{T} Q x}|\hat{y}-y|$, but we also obtain bounds on the maximum error $\delta$ and we can find the image which achieves this maximum error.

\section{CONSTANT Q}

As we mentioned in section two, the choice of $\mathbf{Q}$ is critical. If we assume that our quadratic class is constant over the entire image, we can derive cubic interpolation. We assume that our signals are observed at the output of a filter whose impulse response, in Matlab notation, is $h=$ $[.4830, .8365, .2241,-.1294]$. The filter class

$$
K=\left\{x: x=H u, u \in l_{2},\|u\|_{2} \leq 1\right\}
$$

corresponds to the assumption that all of our signals are modeled as the result of a bounded energy input applied to the filter with unit pulse response $h$. This low-pass $h$ may be familiar; it is the set of scaling equation coefficients corresponding to the length-4 Daubechies wavelet. Suppose we want to increase the sampling rate by a factor of 2 . Specifically, assume that we are given the 4 signal values, $x(-3), x(-1), x(1), x(3)$ and we would like to estimate the center sample, $x(0)$. Since, as shown in [5] the best estimate for signals in $K$ is a linear combination of the representers of the samples, and the representers for the filter class are simply the autocorrelation of the impulse response of the filter, we have representers $\phi$ which are translates by 2 of the autocorrelation

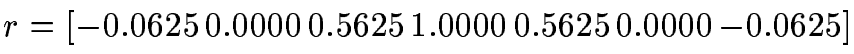

Interpolation with these weights is exactly the same as fitting a cubic polynomial, $p(x)$ to the the 4 known points and using $p(0)$ as the estimate for the sample at index 0 . To estimate any even index sample, given the odd index samples, we simply fit a cubic polynomial to the four points nearest to the sample we want to estimate and then use the value of that polynomial in the center as our estimate. We call this piecewise-cubic interpolation. It is the optimal estimate for

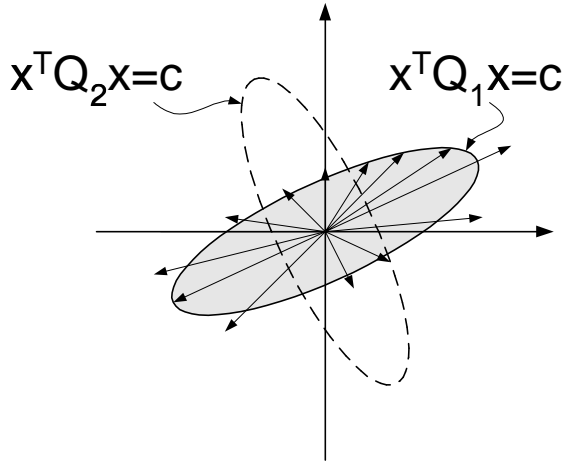

Fig. 1. Training set $S$ and contours corresponding to $x^{T} \mathbf{Q}_{\mathbf{1}} x=c$ and $x^{T} \mathbf{Q}_{\mathbf{2}} x=c$. The training set $S$ is better represented by the contour corresponding to $x^{T} \mathbf{Q}_{\mathbf{1}} x=c$ than the one corresponding to $x^{T} \mathbf{Q}_{\mathbf{2}} x=c$.

this filter class in the sense that the maximum error magnitude

$$
\max _{x \in K}|x(0)-\hat{x}(0)|
$$

is minimized.

\section{LEARNING Q}

In the previous section we discussed an image interpolation example where $\mathbf{Q}$ had been fixed. Better results can be obtained if $\mathbf{Q}$ is learned, adaptively and locally, from the image.

Assume that a given training set $S$ is to be used for learning our matrix $\mathbf{Q}$. For convenience, we further assume that $S$ is a matrix with the training vectors as columns. These training signals lie in a particular region of the space which we assume to be representative of the signal we are trying to interpolate. Our aim is to find a $\mathbf{Q}$ such that the ellipsoid

$$
x^{T} \mathbf{Q} x=\text { constant }
$$

is representative of the training set $S$ as shown in Fig. 1 . What does this mean? We want a $\mathbf{Q}$ for which the contour of $x^{T} \mathbf{Q} x=c$ models the locus of the training set $S$. Intuitively, the contour $x^{T} \mathbf{Q} x=c$ is an ellipsoid stretched in the direction of the eigenvectors of $\mathbf{Q}$. The stretch is largest in the direction where $\mathbf{Q}$ has the smallest eigenvalues and smallest in the direction where $\mathbf{Q}$ has the largest eigenvalues.

The theory of principal components provides us with vectors $\left\{v_{1}, \ldots, v_{n}\right\}$ which minimize the sum of the errors:

$$
E=\sum_{x_{i} \in S}\left\|x_{i}-P_{V}\left(x_{i}\right)\right\|^{2}
$$


where $P_{V}\left(x_{i}\right)$ is the projection of $x_{i}$ on the span of $\left\{v_{1}, \ldots, v_{n}\right\}$. In other words, the principal component vectors are the directions in which the contour $x^{T} \mathbf{Q} x=c$ needs to be stretched. That means that we want them to also be the eigenvectors of matrix $\mathbf{Q}$.

Next, what are the eigenvalues of $\mathbf{Q}$ ? The principal component vectors are the eigenvectors corresponding to the largest eigenvalues of $S S^{T}$. The direction of the set $S$ is that of the eigenvector corresponding to the largest eigenvalue of $S S^{T}$. We want the ellipsoid $x^{T} \mathbf{Q} x=c$ to have the most stretch in that same direction. As mentioned above, the smallest eigenvalues of $\mathbf{Q}$ determine the most stretch in $x^{T} \mathbf{Q} x=c$. Therefore, we want $\mathbf{Q}$ to have small eigenvalues corresponding to the eigenvectors which had large eigenvalues in $S S^{T}$ and $\mathbf{Q}$ to have large eigenvalues corresponding to the eigenvectors which had small eigenvalues in $S S^{T}$. One direct way is to let the eigenvalues of $\mathbf{Q}$ be the reciprocal of the eigenvalues of $S S^{T}$ and the eigenvectors of $\mathbf{Q}$ be the eigenvectors of $S S^{T}$. In other words, $\mathbf{Q}=\left(S S^{T}\right)^{-1}$. If, in constructing $\mathbf{Q}$, we were to consider only the largest principal component and the corresponding eigenvalue, our interpolation result would pretty much simplify to the results of [6].

A alternative motivation for this $\mathbf{Q}$ is the following. Assume that the image pixels of interest, represented as the vector $y$, are a weighted combination of these typical, nearby configurations:

$$
y=S w
$$

where $w$ is the weight vector needed to estimate $y$. We further assume that the simplest explanation of $y$ is the best (Occam's Razor) by assuming that we use the weight vector with minimum sum squared value, i.e. with the minimum of $w^{\prime} w$. This assumption leads us to the requirement on $y$ that $y^{\prime} \tilde{S}^{T} \tilde{S} y$ is minimum, where the matrix $\tilde{S}$ is the pseudo inverse of $S$. This gives us the adaptive quadratic signal class for the pixels on the fine scale:

$$
K=\left\{x \in R^{n}: x^{T} \mathbf{Q} x \leq \epsilon\right\}
$$

where the matrix $\mathbf{Q}=\tilde{S}^{t} \tilde{S}$. This gives the same $\mathbf{Q}$ as the one mentioned previously.

Finally, what's left to do is to choose a training set $S$. It would be nice if the training set could be chosen from the high resolution version of the image, but we can't measure that. Instead, we simply assume that a similar $S$ based on the available coarse scale pixels is a good approximation to our desired $S$. This assumption is similar to that used by $\mathrm{Li}$ [3] in estimating covariance matrices.

\section{RESULTS}

We compared the locally adaptive $\mathbf{Q}$ approach with bicubic (i.e. fixed $\mathbf{Q}$ approach) and fractal-based interpolation [9].
Results can be seen in Fig. 2 and Fig. 3. In both cases, the locally adaptive $\mathbf{Q}$ outperforms the bi-cubic interpolation. We also obtained slightly better results when compared with the methods of [3] and [9]. More results can be found at http://ww.ee.cornell.edu/ ${ }^{\sim}$ splab.

\section{CONCLUSION}

In this paper we presented a new method for image interpolation. The method is based on adaptively determining the quadratic signal class from a set of training vectors and then using optimal recovery to estimate the missing pixels. The method outperforms standard interpolation techniques, such as cubic and linear interpolation. It also seems to fair well against other recently published interpolation methods $[3,9]$.

The quadratic signal class is determined from a set $S$ of training vectors. More work needs to continue on how the training set $S$ is selected adaptively.

\section{REFERENCES}

[1] D. Darian Muresan and Thomas W. Parks, "Adaptive, Optimal-Recovery Image Interpolation," ICASSP 2001, Utah.

[2] D. Darian Muresan and Thomas W. Parks, "Prediction of Image Detail," IEEE ICIP, Vancouver, Sept. 2000 .

[3] Xin Li and Michael T. Orchard, "New Edge Directed Interpolation," IEEE International Conference on Image Processing, Vancouver, Sept. 2000.

[5] Ram G. Shenoy and Thomas W. Parks, "An Optimal Recovery Approach to Interpolation," IEEE Transactions on Signal Processing, Vol. 40, No. 8, (August 1992), pp. 1987-1992.

[6] R. G. Shenoy, N. I. Wijeyesekera, and T. W. Parks, "Interpolation of Transients Based on Training Data," ICASSP, 1991, pp. 1721-1724.

[7] C. A. Michelli and T. J. Rivlin, "A Survey of Optimal Recovery," in Optimal Estimation in Approximation Theory, C. A. Michelli and T. J. Rivlin, Eds. New York: Plenum 1976, pp. 1-54.

[8] M. Golomb and H. F. Weinberger, "Optimal approximation and Error Bounds," On Numerical Approximation, R. E. Langer ed., The University of Wisconsin Press, Maddison, pp. 117-190, 1959.

[9] Commercial, Fractal Based Interpolation Algorithm (www.genuinefractals.com) 

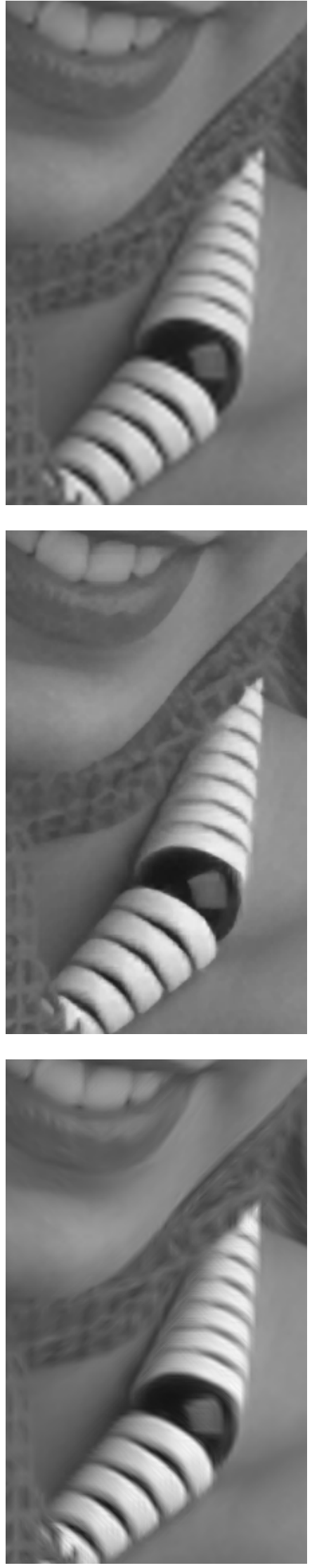

Fig. 2. Interpolation by four. Constant $\mathbf{Q}$ (bi-cubic) (top), [9] (center) and locally adaptive learned $\mathbf{Q}$ (bottom).
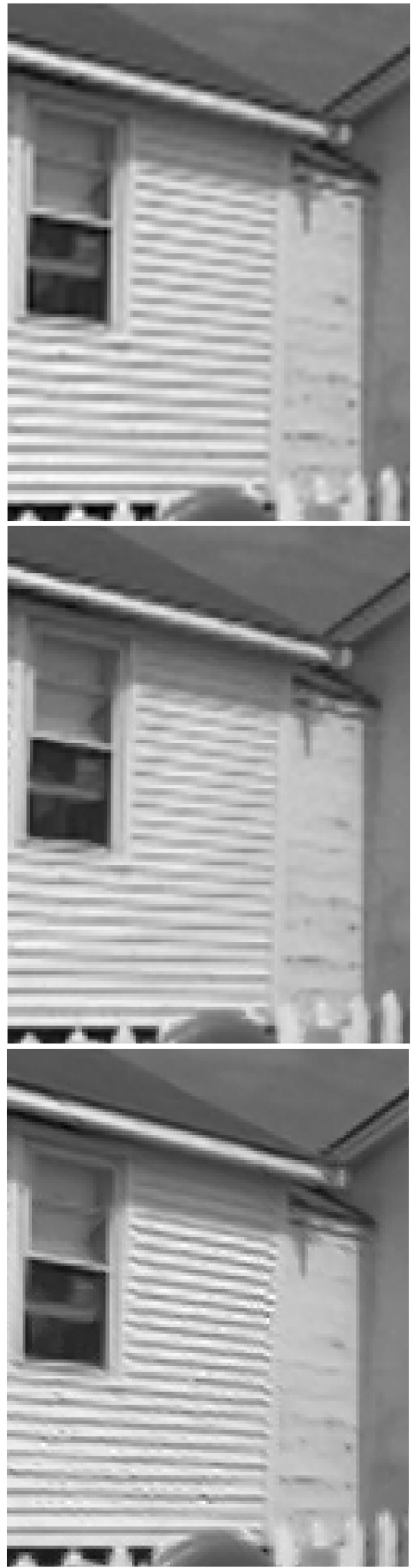

Fig. 3. Interpolation by two. Constant $\mathbf{Q}$ (bi-cubic) (top), [9] (center) and locally adaptive learned $\mathbf{Q}$ (bottom). 